\title{
Rapid Molecular Detection of Tuberculosis and Rifampicine Resistance in Ecuador
}

\author{
Fabián Pardón 1,2, Saddy Andrade², Lilian Campañá4, Homero Jinéz ${ }^{5}$, José P. Barberán' \\ Yolanda Valdés ${ }^{1}$, Alexandra Narváez ${ }^{1}$, Nancy V. Cajas ${ }^{1}$
}

\author{
${ }^{1}$ Faculty of Medical Sciences, University of Specialties Espírito Santo, Guayaquil, Ecuador \\ ${ }^{2}$ Department of Virology, INEI-ANLIS “Dr. Carlos G. Malbrán”, Buenos Aires, Argentina \\ ${ }^{3}$ Laboratory of Bacteriology, Hospital Teodoro Maldonado Carbo, Guayaquil, Ecuador \\ ${ }^{4}$ Faculty of Medicine, Catholic University of Santiago de Guayaquil, Guayaquil, Ecuador \\ ${ }^{5}$ Clinical Laboratory "Dayana", Guayaquil, Ecuador \\ Email: fpardon@hotmail.com, fpardon@anlis.gov.ar
}

How to cite this paper: Pardón, F., Andrade, S., Campañá, L., Jinéz, H., Barberán, J.P., Valdés, Y., Narváez, A. and Cajas, N.V. (2017) Rapid Molecular Detection of Tuberculosis and Rifampicine Resistance in Ecuador. Advances in Infectious Diseases, 7, 126-134.

https://doi.org/10.4236/aid.2017.74013

Received: October 11, 2017

Accepted: November 11, 2017

Published: November 14, 2017

Copyright $\odot 2017$ by authors and Scientific Research Publishing Inc. This work is licensed under the Creative Commons Attribution International License (CC BY 4.0).

http://creativecommons.org/licenses/by/4.0/

\begin{abstract}
Background: In Ecuador, tuberculosis (TB) remains a serious problem that is complicated by the emergence of multidrug-resistant TB (MDR-TB). To evaluate this problem, this study was carried out at the Social Security Hospital (IESS) in Guayaquil, Ecuador from 2013 to 2015. Methods: The Xpert TB/RIF system was used to detect TB and MDR-TB and a survey was carried out to identify the factors that are potentially causing MDR-TB. Findings: $200 \mathrm{~TB}$ patients were confirmed on 5649 suspected patients and 20 (10\%) with MDR-TB. It was observed that the annual prevalence of TB and MDR-TB had declining during study period. Trends have been declining but co-infection has doubled since 2009 with $16 \%$ of patients co-infected with HIV. Potential resistance factors identified were: disruption in drug supply, lack of resources and lack of credibility of treatment.
\end{abstract}

\section{Keywords}

Pulmonary Tuberculosis, HIV, Rifampicin Resistance, GeneXpert

\section{Introduction}

TB is an old disease that is far from being resolved. In fact, in 1993 WHO declared that it was a global emergency. In 2012, there were 9 million TB cases in the world and 1.3 million TB deaths [1], but the problem is more complex because many countries are reporting an increase in multidrug-resistant tuberculosis (MDR-TB) rates. 
MDR-TB was defined as that occurs in TB patients with resistance to the two most effective first-line agents: isoniazid and rifampicin. It was generally estimated in 2014 that MDR-TB represents 3.3\% of new cases and 20\% of retreatment cases [2]. Overall, only $48 \%$ of patients with MDR-TB in the cohort of cases detected in 2010 were treated successfully [1]. It is known that resistance to rifampicin rarely occurs alone and is usually an indicator of resistance to other drugs [3]. In some cases, a more drug-resistant variant of TB may be developed: drug-resistant TB (TB-XDR). XDR-TB is a resistant form with additional resistance to more drugs. It has been reported in 105 countries around the world. On average, it is estimated that $9.6 \%$ of MDR-TB cases may present XDR-TB.

In the Americas, there were 280,000 new cases and 19,000 TB deaths. As indicated in the PAHO/WHO Report of 2014, the estimated incidence rate was 29 cases per 100,000 inhabitants and the case detection rate was 79\% [4]. TB in the Andean region countries is also of concern, as is the case of Colombia where $70 \%$ of patients are cured with supervised treatment, but this is considered a low percentage. Causes of therapeutic failure include non-adherence, lack of medication, and bacterial resistance. Currently, Ecuador has reported an increase in the cure rate that went from $61 \%$ in 2007 to $73 \%$ in 2012 , but it is still a serious problem.

Is it possible that Mycobacterium tuberculosis (MTB) that has been studied since the beginning of microbiology as a science is still a microorganism so difficult to diagnose and treat? Among other causes, the genetic characterization of MTB is the main difficulty, because the MTB genome comprises a wide range of genes with a high GC content and its study will allow a better understanding of the mechanisms of pathogenesis and drug resistance according to Fontalvo et al. [5]. An additional problem is interaction with the human immunodeficiency virus (HIV) since its interaction has led to changes in the epidemiology and clinical aspects of both diseases. MTB facilitates the progression and immune deterioration of the patient with AIDS and HIV in turn to create a favourable environment for the progression of the latent form to the clinical form of TB as referred by Reyes et al. [6] as evidence of this, in 2006 worldwide more than 350 thousand deaths occurred in patients co-infected with HIV [7]. At the same time, TB was the leading cause of death among HIV patients. Based on this situation, $\mathrm{PAHO} / \mathrm{WHO}$ has recommended intensifying the search for $\mathrm{TB}$ cases among people with HIV [4] and to optimize these treatments since anti-TB drugs co-administered with antiretroviral may interact and HIV is constituted in a factor that favours the appearance of resistance as previously reported [6].

In response to these problems in 2010, WHO adopted the $\mathrm{Xpert}^{\circledast} \mathrm{MTB} / \mathrm{RIF}$ system (Cepheid, Sunnyvale, CA, USA). The Xpert ${ }^{\oplus}$ MTB/RIF molecular diagnostic test is being rapidly adopted by countries. By the end of June 2013, 88 of the 145 countries had purchased 1402 for test devices and 3.2 million cartridges with reagents. This assay has shown high sensitivity and specificity as reported by Weyer et al. [8], "For smear-positive culture-positive TB, the pooled sensitivity of Xpert MTB/RIF was 98\% (95\% CrI, 97\% - 99\%) (23 studies, 1952 partici- 
pants) and when used to detect rifampicin resistance, Xpert MTB/RIF achieved a pooled sensitivity of 95\% (95\% CrI, 90\% - 97\%) (17 studies, 555/2624 total specimens) and a pooled specificity of 98\% (95\% CrI, 97\% - 99\%) (24 studies, 2414 specimens, including true negatives and false positives)."

It is an in vitro diagnostic test semi-quantitative PCR, integrated in real time and is based on the simultaneous detection of the MTB gene and rPOB gene mutations associated with RIF resistance, accelerating the diagnosis of TB and MDR-TB. The introduction of Xpert MTB/RIF increased reporting rates for TB cases, as demonstrated by Sachdeva et al. [9] in a study developed in India. In another study conducted in Lima, Peru, Carriquiry et al. [10] reported that MTB/RIF showed excellent performance in patients with advanced immunosuppressant and a high clinical suspicion of TB.

The objective of our study was to evaluate the frequency of TB and MDR-TB in adult patients with high clinical suspicion and to establish the relationship between patients co-infected with HIV and the occurrence of resistance.

\section{Methods}

\subsection{Study Design}

In order to evaluate this problem, a descriptive and transversal study was designed at a hospital in the city of Guayaquil (Ecuador) from 2013 to 2015.

\subsection{Study Population}

Patients older than 18 years with a high clinical suspicion of pulmonary TB who did not receive treatment with anti-TB drugs were included.

A high clinical suspicion of pulmonary TB was defined by Heemskerk et al. [11] as those clinical cases that included cough for ten or more days with abnormal chest $\mathrm{x}$-ray (cavity, focal opacity, pleural effusion, nodule or lymphadenopathy) and at least one of the following symptoms: difficulty respiratory, fever, fatigue, night sweats, haemoptysis, coughing up blood, excessive sweating, especially during the night and chest pain, wheezing or weight loss.

\subsection{Technical Information}

The detection of patients with positive smear microscopy was confirmed by PCR techniques. In addition, we evaluated the frequency of patients co-infected with HIV and resistance to anti-TB drugs.

Patients resistant to rifampin voluntarily agreed to respond to a survey to identify factors that could have contributed to the generation of resistance. An analytical type survey was conducted in face-to-face interviews and a questionnaire with 10 closed questions was used to collect data.

In this study, descriptive statistics were used as a method to describe the variables, using the mean and standard deviation for quantitative variables and frequency and percentage for qualitative variables, which were represented in tables and graphs. All calculations were performed in the statistical program 
SPSS version 22.0 .

All biological samples should be treated with the usual precautions of the health institution. Guidelines for sample management are available from the US Center for Disease Control and Prevention (CDC) [12] and the Clinical and Laboratory Standards Institute (CLSI) [13].

Clinical samples were analyzed using the Xpert MTB/RIF system according the following procedure: $3 \mathrm{ml}$ of sputum were transferred to a $50 \mathrm{ml}$ tube to be decontaminated with $\mathrm{N}$-acetyl-L-cysteine and sodium hydroxide; centrifuge and discard the supernatant and take an aliquot of the decontaminated pellet. $0.5 \mathrm{ml}$ was used for smear staining with Ziehl-Neelsen. For direct MTB/RIF, the sputum sample was carefully mixed to make it homogeneous, then sample reagent was added to $1 \mathrm{ml}$ of untreated sputum on a 2:1 ratio, mixed twice manually during an incubation period of 15 minutes at room temperature, and then $2 \mathrm{ml}$ were transferred to the MTB/RIF cartridge as described in [14].The cartridge was closed and placed into the GeneXpert System.

The GeneXpert system integrates and automates the processing of samples; nucleic acid amplification and detection of target sequences in simple or complex samples. The primers of Xpert MTB/RIF amplify a part of the rPOB gene containing the "core" region of 81 base pairs. Probes can differentiate between natural conserved sequence and mutations in the central region that are associated with resistance.

This system is designed for use only with samples of untreated patients showing clinical signs of TB. The characteristics and method of use were adjusted to the manufacturer's recommendations. The use of Xpert MTB/RIF for the detection of MTB or for determining sensitivity to rifampin has not been validated for patients than are receiving treatment for TB. The study was carried out in a total of 5649 patients with suspected pulmonary TB between January 2013 and June 2015.

\subsection{Ethical Considerations}

The study was approved by the Executive Director of the Hospital. Informed written consent was obtained from all patients included, and their data were treated anonymously, without any bioethical conflicts of any kind.

\section{Results}

As a result, 200 cases were positive for TB and $20(10 \%)$ of them were positive for TB-MR. For diagnosis, 343 smears were analyzed for the presence of MTB and confirmed by PCR. $76 \%$ of TB patients were men with a mean age of 42 years (IQR 28 - 59), 75\% under 60 years and 50\% under 42 years. On the other hand, patients co-infected with HIV have been 32 and represent $16 \%$ of TB positive cases with a male-female ratio of $3.57: 1$ and an average age of 33 years. As mentioned above, patients with MDR-TB were 20 (10\%) and were classified according to sex and HIV co-infection as shown in Table 1. 
Based on these findings, the annual values of Prevalence of TB obtained were $4.45 \%$ (2013); $3.46 \%$ (2014) and 2.42\% (first half of 2015) and resistance values to rifampicin were $0.63 \%$ (2013); $0.33 \%$ (2014) and $0 \%$ (first half of 2015) (Figure 1).

The results of the analytical survey to identify the main factors that may have contributed to the generation of resistance can be seen in Table 2 .

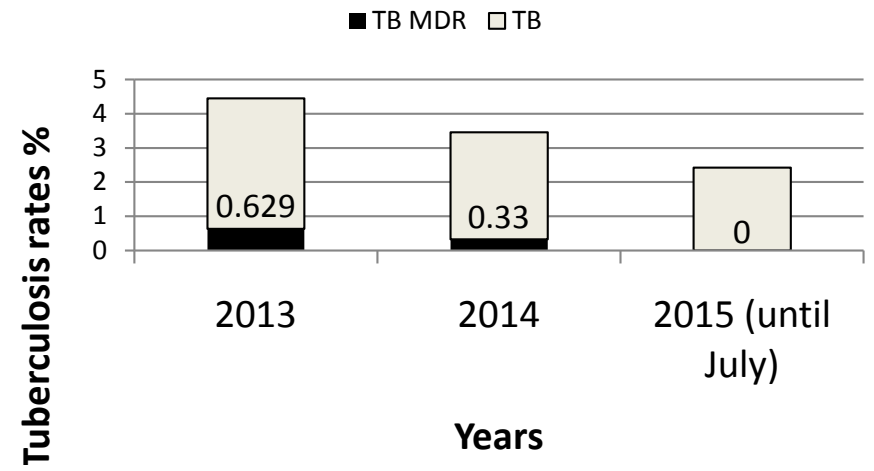

Figure 1. Annual evolution of the rates of TB and MDR-TB expressed in patients with confirmed TB every 100 patients at the Hospital TMC. The prevalence rates of TB and MDR-TB showed a decreasing annual trend consistent with trends reported by Ecuador to WHO.

Table 1. Rifampicin resistant patients classified by sex and HIV coinfection.

\begin{tabular}{cccc}
\hline & HIV negative & HIV positive & Total \\
\hline Female & 4 & 2 & 6 \\
Male & 9 & 5 & $14_{\mathrm{a}}$ \\
Total & 13 & $7_{\mathrm{b}}$ & 20 \\
\hline
\end{tabular}

${ }_{\mathrm{a}} \mathrm{MDR}-\mathrm{TB}$ resistance is more common in men than women at a ratio of 2.3 to $1 ;{ }_{b}$ Seven MDR-TB patients (33\%) had HIV co-infection.

Table 2. Factors that may contribute to the generation of drug resistance.

\begin{tabular}{ccc}
\hline Factor & affirmative & $\%$ \\
\hline Interruption in the supply of drugs & 19 & 95 \\
Lack of resources & 18 & 90 \\
Lack of credibility regarding the effectiveness for treatment & 10 & 50 \\
Messy life style/substance abuse & 9 & 45 \\
Lack of credibility with the diagnosis & 5 & 25 \\
Misunderstanding & 3 & 15 \\
Intolerance/toxicity & 1 & 5 \\
Cultural beliefs & 0 & 0 \\
Pregnancy & 0 & 0 \\
Neuropsychiatric disease & 0 & 0 \\
\hline
\end{tabular}




\section{Discussions}

A perfect correlation (100\%) was observed between Acid fast Bacilli (AFB) and RT-PCR. However, the additional advantage of the molecular technique is that it allows simultaneously diagnose TB and MDR-TB and provides rapid and useful information when evaluating treatments.

Our results over pulmonary TB detection in Ecuador, determined that there is predominance in male patients (3.16 men per woman) and mainly affects the economically active population. Results show an increase compared to previous data, as demonstrated in studies conducted in 2009 where men/women diagnosed with TB positive ratio was 1.7 men for every woman [15].

This value, represents a $62.96 \%$ males and it is according previous reports of WHO worldwide, exists an estimated $60 \%$ of $\mathrm{TB}$ cases are men, a situation that repeats itself when he analyses the TB morbidity and mortality [1]. In relation to age range affected, the National TB Control Program reports [15] that the age range is mainly between 15 and 34 years (men) and coincides with our study ( $50 \%$ of TB cases are under 42 years of age). This finding is worrying because it is an economically active age group and this constitutes an economic problem at the country level.

The study of the prevalence rates, their evolution and the MDR-TB component can be seen in Figure 1, where a decrease in the values of 2014 compared to the year 2013 coincides with the tendency to the low level reported by the WHO [1]. This decline is probably due to the political control of TB that has been implemented without interruption since 2010. The strategy includes the DOTS (Directly Observed Treatment Short Course) program and is generating excellent results in the control of TB as described by Santos-Preciado et al. [16] In 2015 the data are only in the first half and show a tendency to increase the value of the TB rate, but there are no cases of MDR-TB.

The incidence of total TB in Ecuador decreased from 132 in 1990 to 54 in 2014, expressed in cases per 100,000 population, according to World Bank data [17]. Ecuador is the only country in the world that has introduced a subsidy payment that has reduced treatment abandonment from $28.2 \%$ to $7 \%$ in the first year (to ensure compliance with treatment) year of implementation (2011-2012) [1].

Patients with rifampin resistance mutations contributed to the overall indices, as can be seen in Figure 1, where a marked decrease (50\%) was observed in the MDR-TB cases detected from 2013 to 2014. Globally, Proportion of new cases of MDR-TB was 3.5\% in 2013 and has not changed in recent years, approaching the proportional relationship determined in other reports [1]. In $100 \%$ of the patients studied with a confirmed diagnosis of TB, 20 patients (10\%) were found to have resistance mutations.

This value is similar to the resistance values described in previous studies in Ecuador between 2008 and 2009 [14]. Overall, MDR-TB rates were similar to those found in our study [1] [18], it was found that the occurrence of resistance 
is more common in men as can be seen in Table 1 .

Our contribution to the diagnosis of TB cases in the Hospital represents a significant fraction in a district (Guayas) that historically has contributed with $48.25 \%$ of cases throughout the country [14].

A much higher percentage of cases of HIV co-infection have been observed than those reported in studies conducted in 2008 [14], whose values reached $8.8 \%$, whereas in our study HIV patients accounted for $16 \%$. People infected with HIV are 20 times more likely to develop TB than uninfected people. In addition, 7/20 patients with MDR-TB (33\%) had co-infection with HIV (Table 1). Although there are no data in Ecuador on the MDR-TB and HIV ratio, it is significant that the frequency doubles with respect to that detected in TB and HIV co-infection.

\section{Conclusion}

We conclude that although the prevalence rates of TB and MDR-TB shows a decrease, HIV co-infection has doubled since 2009 and this problem is increasing in cases of MDR-TB. We emphasize that TB and MDR-TB affect the male population in the economically active age and the responses of patients with MDR-TB allowed the identification of the main factors that may contribute to the emergence of resistance, the interruption in the supply of drugs, lack of resources $(>90 \%)$ and lack of credibility in the effectiveness or need for treatment $(>50)$.

\section{Acknowledgements}

We thank Dr. Neptali Vera Director of the Hospital Teodoro Maldonado Carbo Bacteriology Laboratory for his assistance during the study and Pedro Sánchez Blanco for the statistical analysis.

\section{Conflict of Interest}

All authors report no conflict of interest in this study.

\section{References}

[1] World Health Organization (2013) Global Tuberculosis Report 2013. http://apps.who.int/iris/bitstream/10665/91355/1/9789241564656_eng.pdf

[2] Trauer, J.M. and Cheng, A.C. (2016) Multidrug-Resistant Tuberculosis in Australia and Our Region. The Medical Journal of Australia, 204, 251-253.

https://doi.org/10.5694/mja16.00012

[3] WHO (2008) Anti-Tuberculosis Drug Resistance in the World Fourth Global Report the WHO/IUATLD Global Project on Anti-Tuberculosis Drug Resistance Surveillance. http://www.who.int/tb/publications/2008/drs_report4_26feb08.pdf

[4] Mitchell, C. Pan American Health Organization/World Health Organization. OPS OMS (2014) La OPS/OMS urge a intensificar acciones para detectar y tratar a las personas coinfectadas con tuberculosis y VIH en las Américas.

http://www.paho.org/hq/index.php?option=com_content\&view=article\&id=9672\% 3Apahowho-calls-for-stepped-up-efforts-to-detect-and-treat-peoplewithtbhivcoinfe 
ctions\&Itemid=1926\&lang=es

[5] Dilia, F.R. and Doris, G.C. (2015) Mycobacterium Tuberculosis Genes Involved in Pathogenicity and Resistance to Antibiotics during Pulmonary and Extrapulmonary Tuberculosis. Medicals UIS, 28, 39-51.

http://www.scielo.org.co/scielo.php?script=sci_arttext\&pid=S0121-03192015000100 $004 \& \operatorname{lng}=\mathrm{en} \& \operatorname{tlng}=\mathrm{es}$

[6] Corcho, A.R. and Jiménez, Y.B. (2003) Tuberculosis in the Context of Acquired Immunodeficiency Syndrome. Overview from the Current Evidence. Revista del Instituto Nacional de Enfermedades Respiratorias, 16, 108-115.

http://www.medigraphic.com/pdfs/iner/in-2003/in032i.pdf

[7] Castiblanco, C.A. and Polpo, C.L. (2008) Tuberculosis in Colombia: Analysis of the Epidemiological Situation, 2006. Infectio, 12, 159-173.

http://www.scielo.org.co/scielo.php?script=sci_arttext\&pid=S0123-93922008000300 002\&lng=en\&tlng

[8] Weyer, K. (2012) Automated Real-Time Nucleic Acid Amplification Technology for Rapid and Simultaneous Detection of Tuberculosis and Rifampicin Resistance: Xpert MTB/RIF System Policy Statement.

http://apps.who.int/iris/handle/10665/112472

[9] Sachdeva, K.S., Raizada, N., Sreenivas, A., Van’T Hoog, A.H., van den Hof, S., Dewan, P.K., Paramasivan, C.N., et al. (2015) Use of Xpert MTB/RIF in Decentralized Public Health Settings and Its Effect on Pulmonary TB and DR-TB Case Finding in India. PLoSOne, 10, e0126065. https://doi.org/10.1371/journal.pone.0126065

[10] Carriquiry, G., Otero, L., González-Lagos, E., Zamudio, C., Sánchez, E., Nabeta, P., Campos, M., Echevarría, J., Seas, C. and Gotuzzo, E. (2012) A Diagnostic Accuracy Study of Xpert ${ }^{\oplus}$ MTB/RIF in HIV-Positive Patients with High Clinical Suspicion of Pulmonary Tuberculosis in Lima, Peru. PLoSOne, 7, e44626.

https://doi.org/10.1371/journal.pone.0044626

[11] Heemskerk, D., Caws, M., Marais, B., et al. (2015) Tuberculosis in Adults and Children. Springer, London, Chapter 3, Clinical Manifestations.

https://www.ncbi.nlm.nih.gov/books/NBK344404/

https://doi.org/10.1007/978-3-319-19132-4

[12] NIAID-NIH (2010) Biosafety in Microbiological and Biomedical Laboratories. 5th Edition. https://www.cdc.gov/biosafety/publications/bmbl5/bmbl.pdf

[13] CLSI (2014) M29-A4: Protection of Laboratory Workers from Occupationally Acquired Infections. 4th Edition, Approved Guideline.

[14] Helb, D., Jones, M., Story, E., Boehme, C., Wallace, E., Ho, K. and Safi, H. (2010) Rapid Detection of Mycobacterium tuberculosis and Rifampin Resistance by Use of On-Demand, Near-Patient Technology. Journal of Clinical Microbiology, 48, 229-237. https://doi.org/10.1128/JCM.01463-09

[15] National Tuberculosis Program (2011) Epidemiological Indicators of Tuberculosis in Ecuador 2000-2009.

http://pctecuador.blogspot.com.ar/2011/03/indicadores-epidemiologicos-ecuador.html

[16] Santos-Preciado, J.I. and Franco-Paredes, C. (2005) Strictly Supervised Shortened Treatment (TAES or DOTS) for Tuberculosis in Populations with Moderate Levels of Drug Resistance: International Impact Perspective. Revista de investigación clínica, 57, 488-490.

http://www.scielo.org.mx/scielo.php?script=sci_arttext\&pid=S0034-8376200500030 $\underline{0013 \& \operatorname{lng}=\mathrm{es} \& \ln \mathrm{l}=\mathrm{es}}$ 
[17] Group, T.W.B. (2016) The World Bank.

http://data.worldbank.org/indicator/SH.TBS.INCD?locations=EC

[18] Bermejo, M.C., Clavera, I., Michel de la Rosa, F.J. and Marín, B. (2007) Epidemiology of Tuberculosis. Anales del sistema sanitario de Navarra Navarre, 30, 7-19. 